Relations industrielles

Industrial Relations

\title{
An International Comparison of Factor Costs and Factor Use, Bagicha Singh Minhas, North-Holland Publishing Company, Amsterdam, 1963, 124 pages.
}

\section{Ronald Pleau}

Volume 20, numéro 4, 1965

URI : https://id.erudit.org/iderudit/027638ar

DOI : https://doi.org/10.7202/027638ar

Aller au sommaire du numéro

Éditeur(s)

Département des relations industrielles de l'Université Laval

ISSN

0034-379X (imprimé)

1703-8138 (numérique)

Découvrir la revue

Citer ce compte rendu

Pleau, R. (1965). Compte rendu de [An International Comparison of Factor Costs and Factor Use, Bagicha Singh Minhas, North-Holland Publishing Company, Amsterdam, 1963, 124 pages.] Relations industrielles / Industrial Relations, 20(4), 723-724. https://doi.org/10.7202/027638ar

Tous droits réservés @ Département des relations industrielles de l'Université Laval, 1965
Ce document est protégé par la loi sur le droit d'auteur. L'utilisation des services d'Érudit (y compris la reproduction) est assujettie à sa politique d'utilisation que vous pouvez consulter en ligne.

https://apropos.erudit.org/fr/usagers/politique-dutilisation/ 
subséquente à cette dernière, la décision et l'cuis de la commission, les témoins et les ossignations, bref le stage formel, qui constituent l'objet d'un quatrième chapitre.

D'autre part lorsque l'on procède d̀ l'étude d'un cos de représentation, les procédures varient quelque peu telles les pétitions (chap. 6) les élections et la durée de l'accréditation (chap. 7).

Enfin dans ce qu'on pourrait appeler une troisième section, il est question de la commission dans ses rapports avec les cours de justice (chap. 8), de la juridiction propre de cette commission d'état et de sa relation ovec la commission nationale connue sous le sigle N.L.R.B. (chap. 9). A tout cela s'ajoute toutes les observations aussi pertinentes que possible qu'effectue Hanslowe concernant la réorganisation administrative de la commission des relations de travail de l'étot de New York et les amendements substantiels qui pourraient être adoptés comme règles du système.

Somme toute le présent exposé semble être des plus utiles comme ouvrage de référence pour qui veut trouver tous les détails concernant la législation ouvrière de cet état américain et la façon de procéder des parties constituantes de son système de relations industrielles en ropport avec l'administration de ses règles.

\section{Ronald Pleau}

\section{Agriculture and the Public Interest Toward} a New Form Program, Leon $H$. Keyserling, Conference on Economic Progress, 1001 Connecticut Avenue, N.W., Washington 6, D.C., 1965, 123 pages.

De foçon générale, l'auteur discute du problème américain de la ferme sous ses aspects domestiques et internationaux, surtout dans sa relation avec l'emploi et les autres problèmes de l'économie en général. II fournit nombre de données statistiques et d'analyses nécessaires à de bonnes recom. mandations politiques sur la question.

Por son étude, il entend saisir les éléments qui, selon lui, offrent la plus grande perspective pour la restauration de l'agriculture américaine. II montre comment les programmes proposés sont adéquats d̀ l'intérieur des ressources nationales disponibles et sensibles aux besoins nationaux et mondioux de ce secteur.

L'étude peut servir à démontrer entre outres choses que les fermiers et leurs organisotions devront faire front commun pour résoudre leur problème de chômage, de sousemploi, etc., en mettant au-dessus de celles qui les divisent les choses qui leur permettent de travailler ensemble. Et pour atteindre ce consensus de travail ils devront recourir à l'aide du monde des affaires, du monde du travail, des consommateurs et du gouvernement.

C'est donc la lutte pour aider les fermiers sous-marginaux à s'élever au-dessus de la pouvreté et devenir des gens qui peuvent se suffire à eux-mêmes, pour rendre les fermes de type familial capables de résister aux accrocs de la grande coopérative agricole, et pour faire en sorte que le maximum de ce que peut produire le fermier américain serve à la rescousse de ceux qui sont présentement affligés par un manque de nutrition, qui constitue l'un des principoux sujets traités dans cet ouvrage.

Pour ce faire, Keyserling a donc choisi de développer les thèmes suivants: le déclin du revenu de la ferme, les causes de ce déclin et les mythes qui sont reliés à ces causes de bas revenus, les raisons véritables de ce bas niveau, l'utilisation plus abondante des produits de la ferme à la maison, le problème mondial du marché de l'alimentation.

Puis passant du domaine plus théorique ou domaine pratique, c'est de la rationalisation de la production agricole et de la restauration du revenu de la ferme dont traite l'étude, pour nous fournir finalement un programme assez élaboré de politiques agricoles ovec ses implications et ses coûts.

Tout ou long de son texte, l'auteur vient appuyer ses affirmations par une série de tableaux statistiques sur la situation agricole aux Etats-Unis, lesquels rehaussent d'autant la valeur du contenu de cet ouvrage.

\section{Ronald Pleau}

\section{An International Comparison of Factor Costs and Factor Use, Bagicho Singh Minhas, North-Holland Publishing Com- pany, Amsterdam, 1963, 124 pages.}

Cette étude fait partie d'une série d'ouvroges en rapport avec l'analyse économique, mais le but spécifique de celle que nous vous présentons ici est de considérer les différences entre les coûts relatifs du travail et du capital pour un groupe de pays, et la facilité ou difficulté relative de substituer l'un pour l'autre dons différentes industries. 
De foçon plus particulière encore l'auteur se propose de faire connaitre certaines relations empiriquement utilisables pour I'analyse de la substitution entre le capital et le travail, de constater, quantitativement, les différences dans le coût du travail et du capital pour un nombre suffisamment considérable d'industries manufacturières et de dériver des estimations numériques d'élasticité de substitution entre ces deux facteurs, et enfin de montrer la signification de ces découvertes empiriques pour les problèmes de commerce international et de développement économique.

Après un premier chapitre dans lequel Minhas expose les données du problème et l'importance de ce dernier, un second nous fournit un cadre théorique pour l'étude des effets des coûts variables d'un focteur sur le modèle d'utilisation de ce dernier.

Puis sur la base d'une régression linéairelogarithmique de la consommation de travail par $\$ 1,000.00$ de valeur ajoutée aux taux de salaires, c'est la dérivation d'estimations de l'élasticité entre le capital et le travail pour vingt-quatre catégories bien définies d'industries monufacturières qui est l'objet de considérations particulières. Les implications de la variabilité de cette substitution sur les avantages comparés eu égard au commerce international sont examinées dans une outre partie de cet exposé.

Dans ces premiers chapitres le toux de rendement du copital joue un rôle d'arrièreplan, voilà pourquoi dans les deux suivants s'opplique-t-on à l'évaluation des différences dans le prix de ce facteur de production pour quelques régions pour lesquelles des données comporables peuvent être obtenues. En examinant le contenu du chapitre six, on remarque que ces estimés de rendement du capitol sont utilisés pour différentes fins comme vérifier l'hypothèse d'une tendance à des rendements égaux dans différents commerces, constater l'efficacité globale du copital industriel entre les pays paurres et les pays riches, et découvrir les différences dans la profitabilité relative de différentes industries dans différents pays.

Enfin, un résumé des principales découvertes de l'étude nous offrant quelques réflexions sur les implications politiques de ces découvertes et certaines suggestions pour des recherches antérieures viennent terminer les propos de l'auteur.

Ronald Pleau

\section{Les installations électriques en moyenne} tension, J. Boulouvard, Entreprise moderne d'édition, Paris, 1964, 78 pages.

Dans son avant-propos, l'auteur avertit le lecteur que son étude a pour but de renseigner les chefs d'étoblissements industriels, militaires et médicaux qui comportent plusieurs bâtiments espocés les uns des autres dans lesquels une instollation électrique est à réaliser en première exécution ou en remplacement d'une ancienne.

L'auteur propose donc d'étudier les installations de moyenne tension (jusqu'd 20,000 volts), qui permettent une distribution d'énergie plus puissante et plus étendue.

Après avoir roppelé l'utilité et l'applicotion de la moyenne tension et les différentes coractéristiques à définir pour son implantotion, l'ouvrage étudie en détail chacune des parties de l'installation à savoir le poste de livraison, le poste de transformation, les côbles, le matériel de comptage, les circuits de terre, les verrouillages et éclairage des locoux.

Suivent ensuite toutes les informations nécessaires à l'installation et d̀ l'exploitotion de ces systèmes telles l'étude et la prévision des incidents électriques dans l'instal'lation, nombre et noture des cellules moyenne-terision, choix du fabricant et des installateurs, implantation des bâtiments, mise en service, entretien, vérification et tarification de ces installations.

Cet ouvrage de défrichement, si on peut dire, puisquaucune documentation complète jusqu'à ce jour n'existoit sur ce sujet, est remarquable par so concision et sa précision. II ne peut qu'être des plus utiles à tous les chefs d'exploitation, les techniciens, les ingénieurs électriciens et les architectes comme ouvrage de bose afin d'ovoir une idée générale concernant ces nouveoux produits le la science.

\section{Ronald Pleau}

Les opprentis scolarisés, par Robert Schiélé et André Moujardet, dans la Collection - L'Enquête et l'Action *, les Editions ouvrières, Paris, 328 pages.

Peu d'enquêtes présentent outant d'intérêt que celle qui nous est préseritée dons cet ouvroge.

A l'origine, quelques apprentis en école veulent sovoir ce que pensent leurs camarades apportenant d̀ d'autres étoblissements. 